\title{
EMPIEMA CRÔNICO COM DRENAGEM ESPONTÂNEA VIA ABDOMINAL
}

\section{CHRONIC EMPYEMA WITH SPONTANEOUS ABDOMINAL DRAINAGE}

Roberto Gibson Ferreira Costa ${ }^{1}$; Breno Machado Costa ${ }^{2}$.

\section{RESUMO}

Introdução: $\mathrm{O}$ empiema torácico é uma entidade que se define pela presença de pus no espaço pleural e apresenta três fases evolutivas bem definidas e com características peculiares. Realizar procedimentos para remover o empiema, reexpandir o pulmão encarcerado, obliterar o espaço pleural e dessa forma restabelecer a mobilidade da parede torácica e do diafragma, e com isso reduzir o tempo de permanência hospitalar, são os objetivos principais no manejo do empiema. Ainda hoje é um grande desafio o tratamento do empiema crônico, principalmente quando associado a lesões de paquipleurite que frequentemente cursam com a formação de cavernas que terminam perpetuando o processo infeccioso e muitas vezes são imperceptiveis durante a cirurgia torácica. Relato do caso: Esta publicação tem como objetivo, apresentar um caso de empiema crônico com 4 anos de evolução que ocupava $2 / 3$ da cavidade pleural esquerda e que se expandia para a cavidade abdominal e evoluiu com drenagem espontânea, além de fazer uma breve revisão literária do assunto. Conclusão: Apesar do avanço tecnológico e da evolução dos antibióticos, os índices de empiema pleural vem aumentando entre adultos e crianças, mantendo uma elevada taxa de mortalidade, por isso o diagnóstico precoce, a distinção das fases evolutivas e a indicação da melhor terapêutica possivel, é a chave para um tratamento bem-sucedido.

Palavras-chave: Empiema Pleural. Infecções Respiratórias. Tuberculose Pulmonar. Cirurgia Torácica.

\section{ABSTRACT}

Introduction: Thoracic empyema is an entity that is defined by the presence of pus in the pleural space and presents three welldefined evolutionary phases with peculiar characteristics. The main objectives in the management of empyema are to perform procedures to remove the empyema, reexpand the incarcerated lung, obliterate the pleural space and thus reestablish the mobility of the chest wall and diaphragm, and reduce the length of hospital stay. The treatment of chronic empyema is still a great challenge, especially when associated to pachypleuritis lesions that frequently course with the formation of cavities that end up perpetuating the infectious process and are many times imperceptible during thoracic surgery. Case report: This paper aims to present a case of chronic empyema with a 4-year evolution that occupied $2 / 3$ of the left pleural cavity and expanded to the abdominal cavity, evolving with spontaneous drainage. Conclusion: Despite technological advances and the evolution of antibiotics, the rates of pleural empyema have been increasing among adults and children, maintaining a high mortality rate, which is why early diagnosis, distinction of the evolutionary phases and indication of the best possible therapy, it is the key to successful treatment.

Keywords: Empyema, Pleural. Respiratory Tract Infections. Tuberculosis, Pulmonary. Thoracic Surgery.

\section{INTRODUÇÃO}

O empiema torácico é uma entidade que se define pela presença de pus no espaço pleural e apresenta três fases evolutivas bem definidas e com características peculiares.

Realizar procedimentos para remover o empiema, reexpandir o pulmão encarcerado, obliterar o espaço pleural e dessa forma restabelecer a mobilidade da parede torácica e do diafragma, e com isso reduzir o tempo de permanência hospitalar, são os objetivos principais no manejo do empiema.

Ainda hoje é um grande desafio o tratamento do empiema crônico, principalmente quando associado a lesões de paquipleurite que frequentemente cursam com a formação de cavernas que terminam perpetuando o processo infeccioso e muitas vezes são imperceptíveis durante a cirurgia torácica.

\footnotetext{
${ }^{1}$ Hospital São Rafael - Monte Tabor - Serviço de Cirurgia Geral - Salvador - BA - Brasil

${ }^{2}$ Hospital São Rafael - Monte Tabor - Serviço de Cirurgia Toracica - Salvador - BA - Brasil
} 
Esta publicação tem como objetivo, apresentar um caso de empiema crônico com 4 anos de evolução que ocupava 2/3 da cavidade pleural esquerda e que se expandia para a cavidade abdominal e evoluiu com drenagem espontânea, além de fazer uma breve revisão literária do assunto.

\section{RELATO DO CASO}

Paciente do gênero masculino, 44 anos, compareceu ao consultório de cirurgia geral com queixa de uma tumoração localizada em fossa ilíaca esquerda com aproximadamente 6 semanas de evolução. $O$ paciente informou que não apresentou perda de peso, não referiu dor no local da tumoração, negou episódios de febre e não associou a lesão a qualquer episodio de trauma no local, mas informou que há aproximadamente 13 anos atrás fez tratamento para Tuberculose Pulmonar.

Ao exame físico, apresentou murmúrio vesicular abolido em $2 / 3$ inferior do hemitorax esquerdo e massa de consistência cística, fixada a planos profundos e indolor a palpação, localizada em fossa iliaca esquerda com cerca de $5,0 \mathrm{~cm}$ em seu maior eixo e sem sinais de flogose no momento da consulta.

Diante disso, foi realizada avaliação laboratorial e de imagem. Os exames laboratoriais não apresentavam alterações significativas, mas os exames de imagem revelaram: volumosa coleção pleural, associada a espessamento e calcificação da pleura à esquerda, promovendo importante efeito compressivo sobre o pulmão adjacente, medindo $21,5 \times 12,0 \times 6,4 \mathrm{~cm}$; localizado na parede anterolateral esquerda, estendendo-se do $1 / 3$ superior ao seio costofrênico lateral do mesmo lado. Observa-se a comunicação da coleção com as partes moles da parede torácica adjacente junto ao seio costofrênico lateral, através de pertuito ao nível do $9^{\circ}$ espaço intercostal, bem como aparente envolvimento do diafragma adjacente, além de brônquios dilatados e tortuosos, com destruição e redução volumétrica do parênquima pulmonar do segmento apicoposterior do lobo superior esquerdo. A coleção se estende pela parede abdominal, através da musculatura e insinua-se na cavidade abdominal, promovendo o espessamento do grande omento com dimensões de $5,1 \times 2,2 \mathrm{~cm}$ e se estendendo até o plano que tangencia a pelve renal esquerda e a loja externa da coleção se estende mais inferiormente com dimensões de $4,8 \times 2,0 \mathrm{~cm}$ (Figura 1).

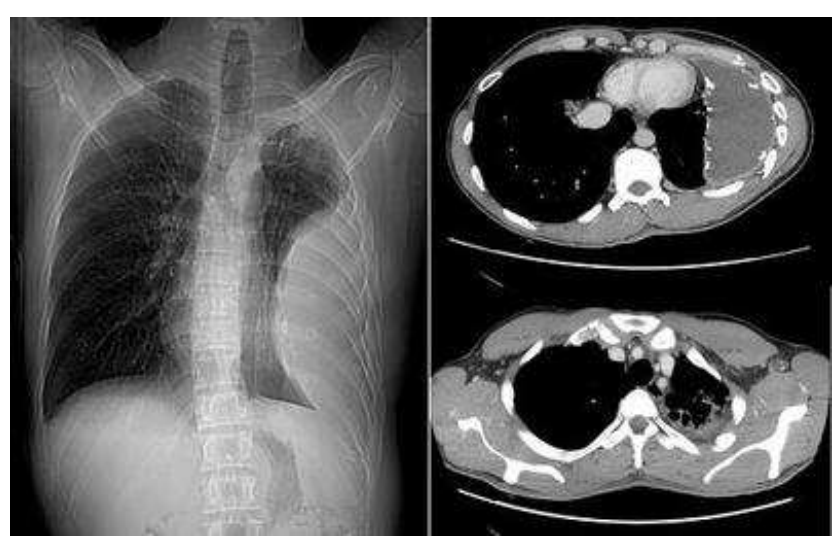

Figura 1: Radiografia e tomografia de tórax apresentando empiema e trajeto fistuloso.

Diante do encontrado foi formulado o diagnóstico de empiema crônico à esquerda com drenagem de necessidade para a fossa iliaca esquerda e o paciente encaminhado ao ambulatório de Cirurgia Torácica.

O paciente foi reavaliado pela Cirurgia Torácica e submetido à toracocentese diagnóstica pré-operatória, colhendo material líquido com característica turva, o qual foi negativo à cultura aeróbia. Foi indicada a realização de pleurostomia com drenagem da coleção abdominal, mas durante a realização dos exames pré-operatórios, o paciente iniciou, em sua residência, a drenagem espontânea da coleção purulenta através da tumoração na fossa ilíaca esquerda, dando entrada na unidade de emergência. Após avaliações de emergência, o paciente foi submetido, no mesmo dia, a drenagem do empiema, coleção de nova amostra do líquido pleural para cultura, biopsia da pleura e confecção de pleurostomia. O paciente evoluiu bem na enfermaria, obtendo alta hospitalar no $5^{\circ}$ DPO.

O exame anatomopatológico da pleura revelou um processo inflamatório crônico com fibrose, consistente com a parede de empiema pleural crônico, não se observando granulomas ou necrose caseosa e a cultura aeróbia foi positiva para Serratia Marcescens.

O paciente vem sendo acompanhado ambulatorialmente e evoluiu bem, sem queixas ou intercorrências, tendo realizado 
tomografias de controle com 11 e 28 meses pós-cirurgia onde se evidencia a redução das dimensões da cavidade pleural e reexpansão lenta e progressiva do parênquima pulmonar com regressão do processo abdominal (Figura 2).

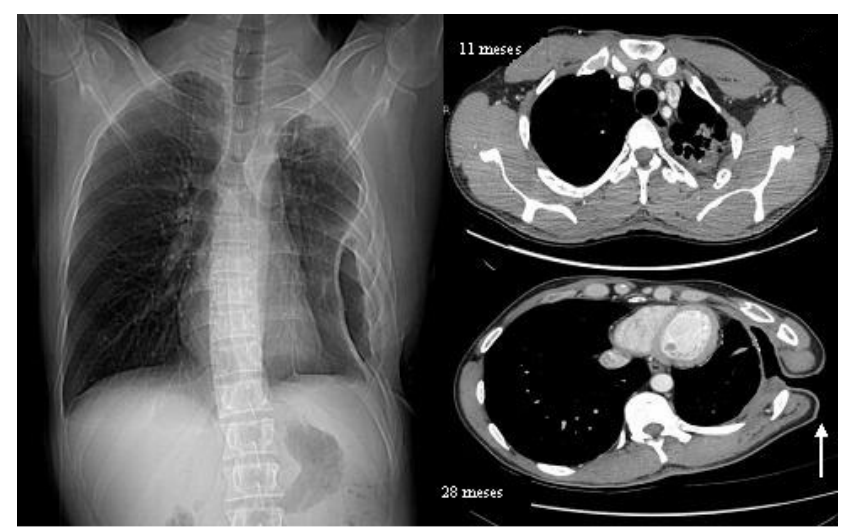

Figura 2: Redução da cavidade pleural e réexpansão do parênquima pulmonar após $11 \mathrm{e}$ 28 meses de pós-operatório.

\section{DISCUSSÃO}

Estudos recentes demonstram um aumento da incidência de empiema tanto entre crianças quanto em adultos e uma mortalidade que varia entre $6 \%$ a $24 \% 1,5$. Apesar de atingir pacientes de todas as idades e classes sociais, o empiema aparece com mais frequência em idosos, pobres e debilitados. Doenças pulmonares crônicas como no caso aqui descrito, neoplasias, doenças cardiacas, diabete, alcoolismo, uso de drogas, neuropatias centrais e imunossupressão, são fatores debilitantes que normalmente estão associados ao empiema pleural.

Os Îndices de mortalidade variam de $1 \%$ a $19 \%$, chegando a ser superior a $40 \%$ em pacientes imunodeprimidos. Geralmente os indivíduos a partir de 50 anos de idade e com comorbidades como: arritmias cardíacas, insuficiência cardíaca congestiva, doenças renais ou doença pulmonar obstrutiva, apresentam maior letalidade e o prognóstico se torna pior quando o paciente desenvolve um empiema secundário a infecções adquiridas em ambiente hospitalar ${ }^{2}$.

Com o passar dos anos, o perfil microbiológico do empiema vem se modificando, provavelmente devido a Era pós-antibioticos, que devido ao uso disseminado e muitas vezes indiscriminado dos antibióticos, foi o responsável pela redução nos indicadores de incidência, morbidade e mortalidade do empiema. O pneumococo e o estafilococo continuam como os principais patógenos e as bactérias aeróbicas gram-negativas e os germes anaeróbios estão cada vez mais envolvidos na etiologia dos empiemas. Já as infecções secundárias por micobactérias e fungos são raras. responsáveis por índices de $20 \%$ a $30 \%$ de empiemas estéreis. De acordo com Bartlett, a baixa incidência de germes anaeróbios, isolados em muitas séries de empiema pleural, decorre dos métodos inadequados de preservação do material (oxigênio sensivel), durante a transferência para o laboratório, além da inexistência de meios de culturas específicos para estes germes em vários laboratórios ${ }^{4,6}$.

O empiema pode ser de causa Traumática ou Não Traumática. As causas traumáticas podem ser divididas em: Iatrogênicas e Não Iatrogênicas. Nas Não Traumáticas se destacam os processos infecciosos torácicos, tendo como principal causa à tuberculose pulmonar como no caso aqui descrito e as supurações pulmonares, além dos extratorácicos que se originam nos quadrantes superiores do abdômen.

$\mathrm{Na}$ década de 60, a "American Thoracic Society" publicou a classificação do empiema em três fases distintas: Aguda, Fibrinopurulenta e Crônica. Essa classificação ao determinar as fases evolutivas do empiema e de suas características, se tornou uma base indispensável para o tratamento desta patologia ${ }^{1,3,5}$.

Fase fibrinopurulenta (transição) Esta fase ocorrera num periodo indefinido de tempo, devido à dependência entre a relação das forças de defesas do organismo versos a virulência do agente causador da infecção. Nesta fase será observado a mudança do líquido pleural limpo e citrino para um líquido mais turvo, onde será visto o aumento progressivo dos valores da DHL, do número de leucócitos e a redução dos niveis de glicose e $\mathrm{pH}$, como resultado do metabolismo bacteriano e fagocitose dos leucócitos. Nesta fase é possivel a identificação do microrganismo agressor $2,4,6$. 
Fase crônica - Se nenhuma ação terapêutica for realizada, em 3 a 4 semanas durante o período da fase fibrinopurulenta, a fase crônica se iniciará. Nesta fase o líquido pleural se tornara espesso e purulento, apresentando niveis baixo de glicose (>40mg/dl) e pH abaixo de 7 , que são características bioquímicas desta fase. A fibrina progressivamente irá sendo substituída por fibroblasto e isso determinará a imobilização do pulmão ou "encarceramento pulmonar"2,4,6.

Os métodos terapêuticos cirúrgicos disponiveis como a toracocentese, drenagem intercostal fechada, pleuroscopia, drenagem aberta, decorticação e toracoplastia, deverão ser indicados para cada fase específica da evolução da doença. A correlação adequada entre os métodos disponiveis e a fase evolutiva, representa o sucesso terapêutico.

$\mathrm{Na}$ fase aguda (exsudativa), a drenagem fechada a selo d'água é a opção mais adequada e na maioria das vezes sob anestesia local. A anestesia geral tem sido reservada para crianças portadoras de empiema4,6.

A fase fibrinopurulenta (transição), abriga o maior número de opções terapêuticas e o que vai caracterizar essa fase será o aparecimento de fibrina e septações pleurais. A drenagem fechada a selo d'água, utilizada como método isolado durante esta fase, tem uma possibilidade considerável de insucesso, com exceção dos casos de urgência no qual o paciente se encontra séptico ou na indisponibilidade de outros recursos cirúrgicos.

$\mathrm{Na}$ intenção de evitar a cronificação do processo infeccioso, nos casos de empiemas que estão na fase inicial e não são resolvidos através da drenagem fechada, a decorticação pulmonar precoce, a pleurectomia parietal e outras técnicas foram propostas como alternativas para os casos de empiema na fase inicial onde não houve sucesso com a drenagem fechada.

A Fase crônica se instala devido a duas circunstâncias: erro no diagnóstico inicial da doença ou fracasso dos métodos terapêuticos utilizados nas fases aguda e fibrinopurulenta. De acordo com algumas pesquisas, a incidência de empiemas parapneumônicos na fase crônica tem declinado, o que mostra uma grande melhora na qualificação do trabalho médico. A fase crônica fica basicamente restrita a drenagem aberta, decorticação pulmonar e a toracoplastia. O procedimento de escolha para esta fase é a drenagem aberta, como indicado e realizado no caso aqui descrito, devido a causar o mínimo de morbidade e mortalidade, além de reduzir o período de hospitalização. Nos casos de insucesso da drenagem fechada, se deve proceder com a pleurostomia para tentar fazer com que o parênquima pulmonar se expanda, permitindo assim uma total ocupação do espaço pleural. Nos casos em que haja a persistência de espaço pleural residual sem possibilidade de expansão, fica indicado as toracoplastias, omentoplastias ou mioplastias, com a intenção de obliteração da cavidade persistente ${ }^{6}$.

A persistência do empiema leva ao aparecimento de sinéquias que obstrui o espaço pleural, praticamente impedindo a sua drenagem e posteriormente levando ao desenvolvimento do fibrotórax ou paquipleurite, que irá impossibilitar a mobilização normal do tórax durante os movimentos respiratórios, provocando uma sindrome restritiva, além da formação de bronquiectasias que resultará numa sindrome ventilatória mista.

No caso aqui apresentado, a primeira cultura do líquido pleural foi negativa, sendo que a positividade da segunda cultura do líquido pleural foi atribuída à contaminação secundária após drenagem espontânea da coleção.

O exame anatomopatológico não revelou granulomas ou necrose caseosa na pleura parietal. Casos como este de "empiema frio" (líquido pleural estéril), e com a drenagem empiemática fazendo-se via abdominal baixa são ainda mais raros.

$$
\text { A despeito das inovações }
$$

tecnológicas e a moderna antibioticoterapia, a incidência de empiema pleural vem aumentando tanto na população adulta quanto na pediátrica, persistindo com elevada taxa de mortalidade. Os aspectos e manifestações clínicas do empiema são diversos. Diagnosticar precocemente, saber distinguir suas fases evolutivas e indicar a melhor opção terapêutica, é a chave para um tratamento bem sucedido. 


\section{REFERÊNCIAS}

1. Gupta R, Crowley S. Increasing paediatric empyema admissions. Thorax. 2006;61(2):179-80. doi: $10.1136 /$ thx.2005.049510.

2. Bender JM, Ampofo K, Sheng X, Pavia AT, Cannon-Albright L, Byington C. Parapneumonic Empyema Death during Past century, Utah. Emerg Infect Dis. 2009;15(1):44-8.

doi: $10.3201 /$ eid1501.080618.

3. Davies CW, Kearney SE, Gleeson FV, Davies RJ. Predictors of outcome and longterm survival in patients with pleural infection. Am J Respir Crit Care Med. 1999;160(5 Pt 1):1682-7. doi: 10.1164/ajrccm.160.5.9903002.

4. Ferguson AD, Prescott RJ, Selkon JB, Watson D, Swinburn CR. Empyema subcommittee of the Research Committee of the British Thoracic Society. The clinical course and management of thoracic empyema. Q J Med. 1996;89:285-9.

5. Mandal AK, Thadepalli H, Mandal AK, Chettipally U. Outcome of primary empyema thoracic: therapeutic and microbiological aspects. Ann Thorac Surg. 1998;66(5):1782-6.

doi: 10.1016/s0003-4975(98)00601-8.

6. Ashbaugh DG. Empyema thoracis. Factors influencing morbidity and mortality. Chest. 1991;99(5):1162-5. doi: $10.1378 /$ chest.99.5.1162.

7. Marchi E, Lundgren F, Mussi R. Parapneumonic effusion and empyema. J Bras Pneumol. 2006;32 Suppl 4:S190-6. doi: 10.1590/S180637132006000900005.
Fonte de financiamento: Não

Conflito de interesses: Não

Data de Submissão: 31 Maio 2021

Decisão final: 26 Stemebro 2021

\section{Autor de Correspondência:}

Roberto Gibson Ferreira Costa

E-mail: gibson660@yahoo.com 